\title{
Medical History End Timepoint
}

National Cancer Institute

\section{Source}

National Cancer Institute. Medical History End Timepoint. NCI Thesaurus. Code C87896.

A point in time that indicates the conclusion of a medical history event. 